\title{
A REMARK ON R. POL'S THEOREM COMCERNING $A$-WEAKLY INFINITE-DIMENSIONAL SPACES
}

\author{
By
}

\author{
Zhu JIAN-PING
}

For notations and relevant definitions we refer to [1].

THEOREM (MA). There is no universal space in the class of all metrizable separable A-weakly infinite-dimensional spaces.

R. Pol proved this theorem in [1] under $C H$. The proof we shall give is similar with the one given in [1] but a little more direct.

LEMMA 1. Let $S \subset I^{\omega}$ be a countable union of zero-dimensional subsets. If $C \subset I^{\omega}$ satisfies that for any open neighbourhood $U$ of $S|C \backslash U|<\mathrm{c}$, then $C \cup S$ is A-weakly infinite-dimensional.

The proof is parallel to the proof of Lemma 1 in [1], noting that in $I^{\omega}$ every subset with cardinality less than $c$ is zero-dimensional.

LEMMA 2 (MA). Let $\left\{G_{\alpha}: \alpha<\lambda\right\}$ be a family of open neighbourhoods of $\Sigma$ in $I^{\omega}$ and $\lambda<\mathrm{c}$, where $\Sigma=\left\{x \in I^{\omega}\right.$ : all but finitely many coordinates of $x$ are equal to zero\}. Then there exist positive numbers $a_{i} \in I(i \in \omega)$ such that $\bigcup_{i}\left[0, a_{i}\right]$ $\subset \cap\left\{G_{\alpha}: \alpha<\lambda\right\}$. Therefore, if $E \subset I^{\omega}$ can be embedded in an A-weakly infinitedimensional space, then $\cap\left\{G_{\alpha}: \alpha<\lambda\right\} \backslash E \neq 0$.

Proof. Let $\mathscr{B}=\{[0,1 / n]: n>0\}$. We define $\boldsymbol{P}=\{(a, b): a$ is a finite sequence in $\left.\mathscr{B} \& b \in[\lambda]^{<\omega}\right\}$ and for any $\left(a^{\prime}, b^{\prime}\right),(a, b) \in \boldsymbol{P}$, where $a=\left(I_{0}, I_{1}, \cdots, I_{n}\right)$ and $a^{\prime}=\left(I_{0}^{\prime}, I_{1}^{\prime}, \cdots, I_{n^{\prime}}^{\prime}\right),\left(a^{\prime}, b^{\prime}\right) \leqq(a, b)$ iff $b^{\prime} \supset b, n \leqq n^{\prime}, I_{i}=I_{i}^{\prime}$ for any $i \leqq n$ and if $n<n^{\prime}, \prod_{i \leqq n} I_{i}^{\prime} \times \prod_{i>n^{\prime}} I \subset \cap\left\{G_{\alpha}: \alpha \in b\right\}$. It is obvious that $\leqq$ is a partial order on $\boldsymbol{P}$. Since all of first components of elements of $\boldsymbol{P}$ are countable, $\boldsymbol{P}$ is $c c c$ (in fact $\sigma$-centred).

Let $D_{\alpha}=\{(a, b) \in \boldsymbol{P}: \alpha \in b\}$ and $F_{n}=\{(a, b)$ : the length of $a$ is larger than $n\}$. It is easily seen that $D_{\alpha}$ is dense in $\boldsymbol{P}$ for any $\alpha<\lambda$. Now we want to

Received September 14, 1989. Revised November 22, 1989. 
show that $F_{n}$ is dense for any $n \in \omega$. Take any $(a, b) \in \boldsymbol{P}$. If the length of $a$ is larger than $n$, then $(a, b) \in F_{n}$. So we suppose that $a=\left(I_{0}, I_{1}, \cdots, I_{m}\right)$, where $m<n$. Since $\cap\left\{G_{\alpha}: \alpha \in b\right\}$ is an open neighbourhood of $\Sigma$, we can find $a^{\prime}=$ $\left(I_{0}, \cdots, I_{m}, I_{m+1}, \cdots, I_{n}\right)$ such that $\prod_{i \leq n} I_{i} \times \prod_{i>n} I \subset \cap\left\{G_{\alpha}: \alpha \in b\right\}$. Therefore, $\left(a^{\prime}, b\right)$ $\leqq(a, b)$ and $\left(a^{\prime}, b\right) \in F_{n}$.

By MA, we have a filter $\boldsymbol{G}$ in $\boldsymbol{P}$ such $\boldsymbol{G} \cap D_{\alpha} \neq 0 \boldsymbol{G} \cap F_{n} \neq 0$ for any $\alpha<\lambda$ and $n<\omega$. Let $\cup\{a$ : there is a $(a, b) \in G\}=\left\{I_{n}: n \in \omega\right\}$. Then $\prod_{n<\omega} I_{n} \subset$ $\cap\left\{G_{\alpha}: \alpha<\lambda\right\}$.

PROOF OF THEOREM. Let $E \subset I^{\omega}$ be any $A$-weakly infinite-dimensional space. Let $\left\{\left(H_{\alpha}, h_{\alpha}\right): \alpha<\mathrm{c}\right\}$ be the family of all pairs such that $H_{\alpha}$ is a $G_{\delta}$-set in $I^{\omega}$ containing $\Sigma$ and $h_{\alpha}: H_{\alpha} \rightarrow I^{\omega}$ is an embedding which maps $\Sigma$ onto a subset of $E$. Let $\left\{G_{\alpha}: \alpha<c\right\}$ be all of the open sets which contain $\Sigma$. Take $x_{\alpha} \in \cap\left\{G_{\beta}: \beta \leqq \alpha\right\} \backslash h_{\alpha}^{-1}(E)$. Then by an argument paralleled to the one in the end of [1], we have $M=\Sigma \cup\left\{x_{\alpha}: \alpha<\mathrm{c}\right\}$ can not be embedded in $E$.

REMARK 3. It is easily seen from the proof of Lemma 2 that the theorem is true under $\mathrm{MA}_{\sigma \text {-centred }}$ i.e. $p=c$, which is strictly weaker than MA.

The author wishes to express his gratitude to Professor Y. Kodama and all of the members in his seminar for their stimulating discusions.

\section{References}

[1] Pol, R., A remark on $A$-weakly infinite-dimensional spaces, Topology and its applications 13 (1982), 97-101.

Zhu Jian-Ping

Institute of Mathematics

University of Tsukuba

Ibaraki 305, Japan 